\title{
OBSERVABILITY OF SQUARE MEMBRANES BY FOURIER SERIES METHODS
}

\section{Komornik, University of Strasbourg, Strasbourg, France,} vilmos.komornik@math.unistra.fr,

P. Loreti, Sapienza University of Rome, Rome, Italy, paola.loreti@sbai.uniroma1.it

Fourier series methods have been successfully applied in control theory for a long time. Some theorems, however, resisted this approach. Some years ago, Mehrenberger succeeded in establishing the boundary observability of vibrating rectangular membranes (and of analogous higher dimensional problems) by developing an ingenious generalization of Ingham's classical theorem on nonharmonic Fourier series. His method turn out to be useful for other applications as well. We improve Mehrenberger's approach by a shorter proof, and we improve and generalize some earlier applications.

Keywords: observability; nonharmonic Fourier series; Ingham's theorem; wave equation.

Dedicated to the memory of Professor Alfredo Lorenzi

\section{Introduction}

Let us consider the wave equation in a bounded domain $\Omega \subset \mathbb{R}^{n}$ with boundary $\Gamma$ :

$$
\begin{cases}u^{\prime \prime}-\Delta u=0 & \text { in } \mathbb{R} \times \Omega, \\ u=0 & \text { on } \mathbb{R} \times \Gamma, \\ u(0)=u_{0} & \text { in } \Omega, \\ u^{\prime}(0)=u_{1} & \text { in } \Omega .\end{cases}
$$

We recall that if $u_{0} \in H_{0}^{1}(\Omega)$ and $u_{1} \in L^{2}(\Omega)$, then the problem has a unique "finiteenergy" solution satisfying

$$
u \in C\left(\mathbb{R} ; H_{0}^{1}(\Omega)\right) \cap C^{1}\left(\mathbb{R} ; L^{2}(\Omega)\right),
$$

and the "energy"

$$
E(t):=\frac{1}{2} \int_{\Omega}|\nabla u(t, x)|^{2}+\left|u^{\prime}(t, x)\right|^{2} d x, \quad t \in \mathbb{R}
$$

of the solution is in fact independent of $t$.

It was discovered by Lasiecka and Triggiani [1] that the normal derivative of finiteenergy solutions is well defined as an element of $L_{\text {loc }}^{2}\left(\mathbb{R} ; L^{2}(\Gamma)\right)$, and for each bounded interval $I$ there exists a constant $c$ such that all finite-energy solutions satisfy the estimate

$$
\int_{I} \int_{\Gamma}\left|\frac{\partial u}{\partial \nu}\right|^{2} d \Gamma d t \leq c E
$$


Lions [2] gave a simpler proof by using the multiplier method.

Subsequently Ho [3] discovered that the inverse inequality also holds if the interval $I$ is long enough. This was improved and generalized by Lions [4,5], who established estimates of the type

$$
E \leq c \int_{I} \int_{S}\left|\frac{\partial u}{\partial \nu}\right|^{2} d \Gamma d t
$$

where $S$ is some part of the boundary $\Gamma$.

Both Ho and Lions applied the multiplier method. By using deeper tools of microlocal analysis, Bardos, Lebeau and Rauch [6] obtained rather precise necessary conditions and sufficient conditions for the validity of such inverse inequalities involving the choice of $S$ and the length of $I$.

Considering the special case where $\Omega \subset \mathbb{R}^{2}$ is a rectangle and $S$ is the union of two adjacent sides, the multiplier method yields the optimal condition: $I$ has to be longer than twice the diagonal $D$ of the rectangle. (In the original proof the optimal condition on $I$ was achieved by a somewhat deeper compactness-uniqueness argument; this was replaced by a short elementary proof in [7].)

Many attempts have been made to recover the last result by Fourier series techniques. Eventually, Mehrenberger [8] succeeded in devising such a proof, although under a stronger condition on $I$ : he needed $|I|>4 \sqrt{2}|D|$ instead of $|I|>2|D|$. His main tool was the following clever generalization of a classical theorem of Ingham on nonharmonic Fourier series:

Theorem 1. Let $\left(\omega_{k}\right)_{k=-\infty}^{\infty}$ be a sequence of real numbers, satisfying for some nonnegative integer $n$ and for some positive real number $\gamma$ the following partial gap condition:

$$
\left|\omega_{k^{\prime}}-\omega_{k}\right| \geq\left|k^{\prime}-k\right| \gamma \quad \text { whenever } \max \left\{\left|k^{\prime}\right|,|k|\right\} \geq n \text {. }
$$

Then the following inequality holds for all square summable sequences $\left(x_{k}\right)_{k=-\infty}^{\infty}$ of complex numbers:

$$
\int_{-R}^{R}\left|\sum_{k=-\infty}^{\infty} x_{k} e^{i \omega_{k} t}\right|^{2} d t \geq \frac{4 R}{\pi}\left(\sum_{|k| \geq n}\left|x_{k}\right|^{2}-\frac{\pi^{2}}{R^{2} \gamma^{2}} \sum_{k=-\infty}^{\infty}\left|x_{k}\right|^{2}\right) .
$$

We remark that the estimate remains valid by translation invariance if we change the integration interval $(-R, R)$ to any other interval of length $2 R$.

This inequality is obvious if $R \leq \frac{\pi}{\gamma}$ because then the right side is nonpositive. For $R>\frac{\pi}{\gamma}$, however, the result is delicate: for $n=0$ this reduces to the deeper part of Ingham's theorem [9].

Theorem 1 proved to be useful for many other control problems as well [10,11].

The plan of this paper is the following:

- For the reader's convenience, in Section 1 we briefly reproduce Mehrenberger's proof of Theorem 1, based on Ingham's first method. ${ }^{1}$

- Applying Ingham's second method, in Section 2 we establish a variant of Theorem 1 , under a weaker condition on $R$.

${ }^{1}$ We recall that Ingham proved his theorem in two different ways. 
- Using the result of Section 2, in Section 3 we weaken the assumption $|I|>4 \sqrt{2}|D|$ for the boundary observability of square membranes to $|I|>4,0441|D|$.

- The method of Section 3 may be applied to improve most results of [10] and [11]. We illustrate this in Section 4 by generalizing the last result of [11], by observing a vibrating rectangular membrane on a finite number of horizontal and vertical lines.

\section{Proof of Theorem 1}

We may assume by scaling that $\gamma>2$ and $R=\pi / 2$.

Following Ingham, we introduce the function $h: \mathbb{R} \rightarrow \mathbb{R}$ and its Fourier transform $H: \mathbb{R} \rightarrow \mathbb{R}$ by the formulas

$$
h(t):= \begin{cases}\cos t & \text { if }|t| \leq \pi / 2 \\ 0 & \text { if }|t| \geq \pi / 2\end{cases}
$$

and

$$
H(x):=\int_{-\infty}^{\infty} h(t) e^{i x t} d t, \quad x \in \mathbb{R} .
$$

If $x \neq \pm 1$, then we have

$$
\begin{aligned}
& H(x)=\int_{-\infty}^{\infty} h(t) e^{i x t} d t=\int_{-\pi / 2}^{\pi / 2} \cos t \cos x t d t=\int_{0}^{\pi / 2} 2 \cos t \cos x t d t= \\
& =\int_{0}^{\pi / 2} \cos (x+1) t+\cos (x-1) t d t=\left[\frac{\sin (x+1) t}{x+1}+\frac{\sin (x-1) t}{x-1}\right]_{0}^{\pi / 2}= \\
& =\frac{\sin (x+1) \frac{\pi}{2}}{x+1}+\frac{\sin (x-1) \frac{\pi}{2}}{x-1}=\frac{\cos \frac{\pi x}{2}}{x+1}-\frac{\cos \frac{\pi x}{2}}{x-1}=\frac{-2 \cos \frac{x \pi}{2}}{x^{2}-1} .
\end{aligned}
$$

Proof of Theorem 1. Since $0 \leq h \leq 1$, we have

$$
\begin{aligned}
& \int_{-\pi / 2}^{\pi / 2}\left|\sum_{k=-\infty}^{\infty} a_{k} e^{i \omega_{k} t}\right|^{2} d t \geq \int_{-\infty}^{\infty} h(t)\left|\sum_{k=-\infty}^{\infty} a_{k} e^{i \omega_{k} t}\right|^{2} d t=\sum_{\max \left\{\left|k^{\prime}\right|,|k|\right\} \geq n} H\left(\omega_{k^{\prime}}-\omega_{k}\right) a_{k}^{\prime} \overline{a_{k}}+ \\
& +\int_{-\infty}^{\infty} h(t)\left|\sum_{|k|<n} a_{k} e^{i \omega_{k} t}\right|^{2} d t \geq \sum_{\max \left\{\left|k^{\prime}\right|,|k|\right\} \geq n} H\left(\omega_{k^{\prime}}-\omega_{k}\right) a_{k}^{\prime} \overline{a_{k}}=H(0) \sum_{|k| \geq n}\left|a_{k}\right|^{2}+ \\
& +\sum_{\substack{\max \left\{\left|k^{\prime}\right|,|k|\right\} \geq n \\
k^{\prime} \neq k}} H\left(\omega_{k^{\prime}}-\omega_{k}\right) a_{k}^{\prime} \overline{a_{k}} \geq H(0) \sum_{|k| \geq n}\left|a_{k}\right|^{2}-\sum_{\substack{\max \left\{\left|k^{\prime}\right|,|k|\right\} \geq n \\
k^{\prime} \neq k}}\left|H\left(\omega_{k^{\prime}}-\omega_{k}\right)\right| \frac{\left|a_{k}\right|^{2}+\left|a_{k^{\prime}}\right|^{2}}{2} .
\end{aligned}
$$

Since the function $H$ is even, the last expression does not change if we change the last fraction to $\left|a_{k}\right|^{2}$. Distinguishing in the last sum the cases $|k| \geq n$ and $|k|<n$, it follows 
that

$$
\begin{aligned}
\int_{-\pi / 2}^{\pi / 2}\left|\sum_{k=-\infty}^{\infty} a_{k} e^{i \omega_{k} t}\right|^{2} d t & \geq \\
& \geq \sum_{|k| \geq n}\left|a_{k}\right|^{2}\left(H(0)-\sum_{k^{\prime} \neq k}\left|H\left(\omega_{k^{\prime}}-\omega_{k}\right)\right|\right)- \\
& -\sum_{|k|<n}\left|a_{k}\right|^{2} \sum_{\left|k^{\prime}\right| \geq n}\left|H\left(\omega_{k^{\prime}}-\omega_{k}\right)\right| .
\end{aligned}
$$

The proof will be completed if we show the following three relations:

$$
\begin{aligned}
& H(0)=2 \\
& \sum_{k^{\prime} \neq k}\left|H\left(\omega_{k^{\prime}}-\omega_{k}\right)\right| \leq \frac{8}{\gamma^{2}} \quad \text { if } \quad|k| \geq n ; \\
& \sum_{\left|k^{\prime}\right| \geq n}\left|H\left(\omega_{k^{\prime}}-\omega_{k}\right)\right| \leq \frac{8}{\gamma^{2}} \quad \text { if } \quad|k|<n .
\end{aligned}
$$

The first equality follows at once from the explicit expression of $H(x)$. The second and third relations also follow from this expression and from the gap condition $\gamma>2$. Indeed, we have

$$
|H(x)| \leq \frac{2}{x^{2}-1}
$$

if $|x|>1$. Since $\gamma>2$, in case $|k| \geq n$ we have

$$
\begin{aligned}
& \sum_{k^{\prime} \neq k}\left|H\left(\omega_{k^{\prime}}-\omega_{k}\right)\right| \leq \sum_{k^{\prime} \neq k} \frac{2}{\left|\omega_{k^{\prime}}-\omega_{k}\right|^{2}-1} \leq \sum_{k^{\prime} \neq k} \frac{2}{\left|k^{\prime}-k\right|^{2} \gamma^{2}-1} \leq \sum_{k^{\prime} \neq k} \frac{2}{\left|k^{\prime}-k\right|^{2} \gamma^{2}-\frac{\gamma^{2}}{4}} \\
& =\frac{8}{\gamma^{2}} \sum_{k^{\prime} \neq k} \frac{1}{4\left|k^{\prime}-k\right|^{2}-1}=\frac{8}{\gamma^{2}} \sum_{m=1}^{\infty} \frac{2}{4 m^{2}-1}=\frac{8}{\gamma^{2}} \sum_{m=1}^{\infty}\left(\frac{1}{2 m-1}-\frac{1}{2 m+1}\right)=\frac{8}{\gamma^{2}} .
\end{aligned}
$$

The proof of the third relation is identical.

\section{A Variant of Mehrenberger's Ingham Type Theorem}

Given two positive numbers $\gamma$ and $R$, we introduce four continuous even functions $H, G, h, g: \mathbb{R} \rightarrow \mathbb{R}$ by the following formulas:

$$
\begin{aligned}
H(x) & := \begin{cases}\cos \frac{\pi x}{\gamma} & \text { if } x \leq \gamma / 2 \\
0 & \text { if } x \geq \gamma / 2\end{cases} \\
G:= & R^{2}(H * H)+H^{\prime} * H^{\prime} \\
h(t):= & \int_{-\infty}^{\infty} H(x) e^{i t x} d x \\
g(t):= & \int_{-\infty}^{\infty} G(x) e^{i t x} d x
\end{aligned}
$$


We are going to prove the following variant of Theorem 1:

Theorem 2. Let $\left(\omega_{k}\right)_{k=-\infty}^{\infty}$ be a sequence of real numbers, satisfying for some nonnegative integer $n$ and for some positive real number $\gamma$ the partial gap condition

$$
\left|\omega_{k^{\prime}}-\omega_{k}\right| \geq\left|k^{\prime}-k\right| \gamma \quad \text { whenever } \max \left\{\left|k^{\prime}\right|,|k|\right\} \geq n \text {. }
$$

Then the following inequality holds for all square summable sequences $\left(x_{k}\right)_{k=-\infty}^{\infty}$ of complex numbers:

$$
\int_{-R}^{R}\left|\sum_{k=-\infty}^{\infty} x_{k} e^{i \omega_{k} t}\right|^{2} d t \geq \frac{2 \pi}{\max |g|}\left(G(0) \sum_{k=-\infty}^{\infty}\left|x_{k}\right|^{2}-\max |G| \sum_{|k|<n}\left|x_{k}\right|^{2}\right) .
$$

We notice that

$$
\alpha:=\max |g|=\max _{[-R, R]} g<\infty \quad \text { and } \quad \beta:=\max |G|=\max _{[-\gamma, \gamma]}|G|<\infty .
$$

The first relation follows from the formula $g(t)=\left(R^{2}-t^{2}\right) h(t)^{2}$ implying that $g \geq 0$ in $[-R, R]$ and $g \leq 0$ outside $[-R, R]$. The second one holds because $G$ vanishes outside $[-\gamma, \gamma]$.

Since $g$ and $G$ do not vanish identically, we have also $\alpha, \beta>0$.

Proof. We write

$$
x(t):=\sum_{k=-\infty}^{\infty} a_{k} e^{i \omega_{k} t}
$$

for brevity.

Using the Fourier inversion formula

$$
\int_{-\infty}^{\infty} g(t) e^{-i t x} d t=2 \pi G(x)
$$

we have

$$
\alpha \int_{-R}^{R}|x(t)|^{2} d t \geq \int_{-\infty}^{\infty} g(t)|x(t)|^{2} d t=2 \pi \sum_{k, k^{\prime}=-\infty}^{\infty} G\left(\omega_{k}-\omega_{k^{\prime}}\right) x_{k} \overline{x_{k^{\prime}}} .
$$

Since $G=0$ outside $[-\gamma, \gamma]$, applying (3) the last sum is equal to

$$
G(0) \sum_{k=-\infty}^{\infty}\left|x_{k}\right|^{2}+\sum_{\substack{|k|<n \\\left|k^{\prime}\right|<n \\ k \neq k^{\prime}}} G\left(\omega_{k}-\omega_{k^{\prime}}\right) x_{k} \overline{x_{k^{\prime}}}
$$

Remarking that

$$
\sum_{\substack{|k|<n \\\left|k^{\prime}\right|<n \\ k \neq k^{\prime}}} G\left(\omega_{k}-\omega_{k^{\prime}}\right) x_{k} \overline{x_{k^{\prime}}} \geq-\beta \sum_{\substack{|k|<n \\\left|k^{\prime}\right|<n}} \frac{\left|x_{k}\right|^{2}+\left|x_{k^{\prime}}\right|^{2}}{2}=-\beta \sum_{|k|<n}\left|x_{k}\right|^{2}
$$


we deduce from (5) the inequality

$$
\int_{-R}^{R}|x(t)|^{2} d t \geq \frac{2 \pi}{\alpha}\left(G(0) \sum_{k=-\infty}^{\infty}\left|x_{k}\right|^{2}-\beta \sum_{|k|<n}\left|x_{k}\right|^{2}\right)
$$

i.e., (4).

Remark 1. For the purposes of the next section we determine explicitly the function $G$. For $\gamma=\pi$ an easy computation leads to the expressions ${ }^{2}$

$$
g(t)=\tilde{g}(t):=\frac{4\left(R^{2}-t^{2}\right)}{(1+t)^{2}(1-t)^{2}} \cos ^{2} \frac{\pi t}{2}
$$

and

$$
2 G(x)=\tilde{G}(x):= \begin{cases}\left(R^{2}+1\right) \sin x+\left(R^{2}-1\right)(\pi-x) \cos x, & \text { if } x \leq \pi \\ 0, & \text { if } x \geq \pi\end{cases}
$$

The general case hence follows by a change of variable. ${ }^{3}$ Setting $c:=\pi / \gamma$ we have $H(x)=\tilde{H}(c x)$,

$$
\begin{aligned}
& h(t)=\int H(x) e^{i t x} d x=\int \tilde{H}(c x) e^{i(t / c)(c x)} d x=c^{-1} \int \tilde{H}(c x) e^{i(t / c)(c x)} d(c x)=c^{-1} \tilde{h}(t / c) \\
& G(x)=R^{2}(H * H)(x)+\left(H^{\prime} * H^{\prime}\right)(x)=R^{2} \int H(y) H(x-y) d y+ \\
& +\int H^{\prime}(y) H^{\prime}(x-y) d y=R^{2} \int \tilde{H}(c y) \tilde{H}(c x-c y) d y+c^{2} \int \tilde{H}^{\prime}(c y) \tilde{H}^{\prime}(c x-c y) d y= \\
& =c^{-1} R^{2} \int \tilde{H}(z) \tilde{H}(c x-z) d z+c \int \tilde{H}^{\prime}(z) \tilde{H}^{\prime}(c x-z) d z \\
& =c\left(\frac{R^{2}}{c^{2}}(\tilde{H} * \tilde{H})+\left(\tilde{H}^{\prime} * \tilde{H}^{\prime}\right)\right)(c x) ; \\
& g(t)=\int G(x) e^{i t x} d x=c^{-1} R^{2} \int(\tilde{H} * \tilde{H})(c x) e^{i t x} d x+c \int\left(\tilde{H}^{\prime} * \tilde{H}^{\prime}\right)(c x) e^{i t x} d x= \\
& =c^{-1} R^{2} \int(\tilde{H} * \tilde{H})(c x) e^{i(t / c)(c x)} d x+c \int\left(\tilde{H}^{\prime} * \tilde{H}^{\prime}\right)(c x) e^{i(t / c)(c x)} d x= \\
& =c^{-2} R^{2} \int(\tilde{H} * \tilde{H})(z) e^{i(t / c) z} d z+\int\left(\tilde{H}^{\prime} * \tilde{H}^{\prime}\right)(z) e^{i(t / c) z} d z= \\
& =c^{-2} R^{2} \int(\tilde{H} * \tilde{H})(z) e^{i(t / c) z} d z-c^{-2} t^{2} \int\left(\tilde{H}^{2} * \tilde{H}\right)(z) e^{i(t / c) z} d z=c^{-2}\left(R^{2}-t^{2}\right) \tilde{h}^{2}(t / c) .
\end{aligned}
$$

${ }^{2}$ See [12, pp. 62 and 64.].

${ }^{3}$ All integrals will be taken over $\mathbb{R}$. 


\section{Boundary Observability of Square Membranes I}

We investigate the validity of the inverse inequality (2) for the solutions of (1) when $\Omega$ is a square membrane with diagonal $D$. (The computations of this section may be easily adapted to general rectangular domains but the results are less elegant.)

We are going to prove the following

Proposition 1. If $|I|>4,0441|D|$, then the finite energy solutions of (1) satisfy the estimate (2).

Since our assumption is between $|I|>4 \sqrt{2}|D|$ and the optimal condition $|I|>2|D|$, our result is weaker than the result obtained by the multiplier method, but stronger than the first theorem found in [8] by Fourier series techniques.

Remark 2. As in [8], the proof of the proposition, given below, may be adapted to higher dimensions when $\Omega$ is an $N$-dimensional interval, and $S$ is the union of the sides of $\Omega$ having a common vertex. Furthermore, we may consider various other (mixed) boundary conditions such that the corresponding eigenfunctions are still products of sine and cosine functions.

For the proof we assume by scaling that $\Omega=(0, \pi) \times(0, \pi)$ and

$$
S:=\left\{\left(x_{1}, x_{2}\right) \in[0, \pi] \times[0, \pi]: x_{1}=0 \text { or } x_{2}=0\right\}
$$

is the union of two adjacent sides.

We recall that the solutions of (1) are given by the series

$$
u\left(t, x_{1}, x_{2}\right)=\sum_{k_{1}=1}^{\infty} \sum_{k_{2}=1}^{\infty}\left(a_{k} e^{i|k| t}+b_{k} e^{-i|k| t}\right) \sin \left(k_{1} x_{1}\right) \sin \left(k_{2} x_{2}\right),
$$

with the usual notation $|k|:=\sqrt{k_{1}^{2}+k_{2}^{2}}$, and that the complex coefficients satisfy the equality (see, e.g., [11, (3.7)])

$$
E=\frac{\pi^{2}}{4} \sum_{k_{1}=1}^{\infty} \sum_{k_{2}=1}^{\infty}|k|^{2}\left(\left|a_{k}\right|^{2}+\left|b_{k}\right|^{2}\right) .
$$

Turning to the study of the boundary integral first we observe that

$$
\begin{aligned}
& \int_{-R}^{R} \int_{0}^{\pi}\left|\frac{\partial u}{\partial x_{2}}\left(t, x_{1}, 0\right)\right|^{2} d x_{1} d t= \\
&=\int_{-R}^{R} \int_{0}^{\pi}\left|\sum_{k_{1}=1}^{\infty} \sum_{k_{2}=1}^{\infty} k_{2}\left(a_{k} e^{i|k| t}+b_{k} e^{-i|k| t}\right) \sin \left(k_{1} x_{1}\right)\right|^{2} d x_{1} d t= \\
&=\frac{\pi}{2} \sum_{k_{1}=1}^{\infty} \int_{-R}^{R}\left|\sum_{k_{2}=1}^{\infty}\left(k_{2} a_{k} e^{i|k| t}+k_{2} b_{k} e^{-i|k| t}\right)\right|^{2} d t .
\end{aligned}
$$

We need the following lemma: 
Lemma 1. Fix an integer $N \geq 2$ and $N-1$ integers $k_{1}, \ldots, k_{N-1} \geq 1$. If $k_{N}, k_{N}^{\prime}$ are two positive integers satisfying

$$
\max \left\{k_{N}, k_{N}^{\prime}\right\} \geq \max \left\{k_{1}, \ldots, k_{N-1}\right\},
$$

then

$$
\left|\sqrt{k_{1}^{2}+\cdots+k_{N-1}^{2}+k_{N}^{2}}-\sqrt{k_{1}^{2}+\cdots+k_{N-1}^{2}+\left(k_{N}^{\prime}\right)^{2}}\right| \geq(\sqrt{N}-\sqrt{N-1})\left|k_{N}-k_{N}^{\prime}\right| .
$$

This lemma improves a result in [8] by increasing the constant $1 / 2 \sqrt{N}$ to $\sqrt{N}-$ $\sqrt{N-1}$. For example, we get $\sqrt{2}-1 \approx 0,41$ instead of $1 / 2 \sqrt{2} \approx 0,35$ if $N=$ Pr:oof. Assuming by symmetry that $k_{N}>k_{N}^{\prime}$ and setting $s:=k_{1}^{2}+\cdots+k_{N-1}^{2}$ for brevity, we have $s \leq(N-1) k_{N}^{2}$, and

$$
\begin{aligned}
\frac{\left|\sqrt{s+k_{N}^{2}}-\sqrt{s+\left(k_{N}^{\prime}\right)^{2}}\right|}{\left|k_{N}-k_{N}^{\prime}\right|} & =\frac{\sqrt{s+k_{N}^{2}}-\sqrt{s+\left(k_{N}^{\prime}\right)^{2}}}{k_{N}-k_{N}^{\prime}}= \\
& =\frac{k_{N}+k_{N}^{\prime}}{\sqrt{s+k_{N}^{2}}+\sqrt{s+\left(k_{N}^{\prime}\right)^{2}}} \geq \frac{k_{N}+k_{N}^{\prime}}{\sqrt{N k_{N}^{2}}+\sqrt{(N-1) k_{N}^{2}+\left(k_{N}^{\prime}\right)^{2}}} .
\end{aligned}
$$

Setting $x:=k_{N}^{\prime} / k_{N} \in(0,1)$ for brevity, the last expression is equal to

$$
f(x):=\frac{1+x}{\sqrt{N}+\sqrt{N-1+x^{2}}} .
$$

The lemma follows because $f(x)>f(0)=\sqrt{N}-\sqrt{N-1}$ for all $x \in(0,1)$.

Indeed, $f$ is strictly increasing because

$$
f^{\prime}(x)=\frac{1}{\left(\sqrt{N}+\sqrt{N-1+x^{2}}\right)^{2}}\left(\sqrt{N}+\frac{N-1-x}{\sqrt{N-1+x^{2}}}\right)>0
$$

for all $x \in(0,1)$.

Using the case $N=2$ of this lemma we may estimate the last integrals in (8) by applying Theorem 2 with $n=k_{1}$ and $\gamma=\sqrt{2}-1$ for each fixed $k_{1}$. This yields the following inequality:

$$
\begin{aligned}
& \frac{\alpha}{\pi^{2}} \int_{-R}^{R} \int_{0}^{\pi}\left|\frac{\partial u}{\partial x_{2}}\left(t, x_{1}, 0\right)\right|^{2} d x_{1} d t \geq \\
& \geq G(0) \sum_{k_{1}=1}^{\infty} \sum_{k_{2}=1}^{\infty} k_{2}^{2}\left(\left|a_{k}\right|^{2}+\left|b_{k}\right|^{2}\right)-\beta \sum_{k_{1}=1}^{\infty} \sum_{k_{2}<k_{1}} k_{2}^{2}\left(\left.a_{k}\right|^{2}+\left|b_{k}\right|^{2}\right) \geq \\
& \geq G(0) \sum_{k_{1}=1}^{\infty} \sum_{k_{2}=1}^{\infty} k_{2}^{2}\left(\left|a_{k}\right|^{2}+\left|b_{k}\right|^{2}\right)-\frac{\beta}{2} \sum_{k_{1}=1}^{\infty} \sum_{k_{2}<k_{1}}|k|^{2}\left(\left|a_{k}\right|^{2}+\left|b_{k}\right|^{2}\right) .
\end{aligned}
$$


By symmetry we also have

$$
\begin{aligned}
\frac{\alpha}{\pi^{2}} \int_{-R}^{R} \int_{0}^{\pi}\left|\frac{\partial u}{\partial x_{1}}\left(t, 0, x_{2}\right)\right|^{2} d x_{2} d t & \geq \\
& \geq G(0) \sum_{k_{1}=1}^{\infty} \sum_{k_{2}=1}^{\infty} k_{1}^{2}\left(\left|a_{k}\right|^{2}+\left|b_{k}\right|^{2}\right)-\frac{\beta}{2} \sum_{k_{2}=1}^{\infty} \sum_{k_{1}<k_{2}}|k|^{2}\left(\left|a_{k}\right|^{2}+\left|b_{k}\right|^{2}\right) .
\end{aligned}
$$

Adding the two inequalities we conclude that

$$
\int_{-R}^{R} \int_{S}\left|\frac{\partial u}{\partial \nu}\right|^{2} d \Gamma d t \geq \frac{\pi^{2}}{\alpha}\left(G(0)-\frac{\beta}{2}\right) \sum_{k_{1}=1}^{\infty} \sum_{k_{2}=1}^{\infty}|k|^{2}\left(\left|a_{k}\right|^{2}+\left|b_{k}\right|^{2}\right) .
$$

Taking (7) into account we obtain our final estimate:

$$
\int_{-R}^{R} \int_{S}\left|\frac{\partial u}{\partial \nu}\right|^{2} d \Gamma d t \geq \frac{4 G(0)-2 \beta}{\alpha} E .
$$

It remains to choose $R>0$ so as to satisfy $4 G(0)-2 \beta>0$, i.e.,

$$
\max G<2 G(0) \text {. }
$$

In view of the last remark of the preceding section this is equivalent to the inequality

$$
(K+1) \sin x+(K-1)(\pi-x) \cos x<2(K-1) \pi,
$$

for all $0<x \leq \pi$, where we write $K:=R^{2} \gamma^{2} / \pi^{2}$ for brevity. This is equivalent to

$$
\frac{K+1}{K-1}<\frac{2 \pi+(x-\pi) \cos x}{\sin x}
$$

for all $0<x \leq \pi$, and this is satisfied by a simple computation if $\frac{K+1}{K-1}<5,97$, which in turn is satisfied if $K>1,403$. This is equivalent to ${ }^{4}$

$$
R>\sqrt{1,403} \pi / \gamma \approx 1,18448301 \pi / \gamma .
$$

Using the value $\gamma=\sqrt{2}-1$ we arrive finally to the sufficient condition $R>2,859594947 \pi$ of the proposition.

\section{Internal Observability of Square Membranes}

We continue to consider sufficiently smooth solutions of (3). The following notion was introduced in [13] and generalized in [11]:

\footnotetext{
${ }^{4}$ This is better than the condition $R>\sqrt{2} \pi / \gamma$ in [8].
} 
Definition 1. A function $f \in L^{1}(0, \pi)$ is $p$-cyclic for some integer $p \geq 2$ if its $2 \pi$-periodic odd extension satisfies the equality

$$
\sum_{k=1}^{p} f\left(t+\frac{2 k \pi}{p}\right)=0
$$

for almost all $t \in \mathbb{R}$.

We fix two integers $p, q \geq 2$ and we consider only smooth solutions of (3) whose initial data are $p$-cyclic in the first variable $x_{1}$ and $q$-cyclic in the second variable $x_{2}$. We observe the solutions simultaneously on $p-1$ vertical lines and $q-1$ horizontal lines, given by the equations

$$
x_{1}=\frac{j \pi}{p}, \quad j=1, \ldots, p-1 \quad \text { and } \quad x_{2}=\frac{j \pi}{q}, \quad j=1, \ldots, q-1 .
$$

We call these solutions $(p, q)$-cyclic; see the figure below.

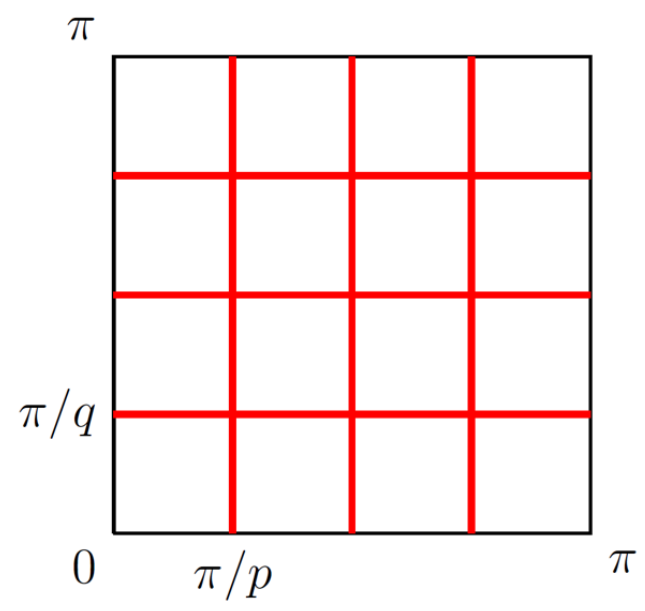

Our purpose is to prove the following theorem:

Theorem 3. If $p=q$ and

$$
R>\frac{(2+\sqrt{2}) \pi}{p}
$$

then there exists a positive constant $c=c(R)$ such that all $(p, q)$-cyclic solutions of $(1)$ satisfy the inequality

$$
c E \leq \sum_{j=1}^{p-1} \int_{-R}^{R} \int_{0}^{\pi}\left|u^{\prime}\left(t, \frac{j \pi}{p}, z\right)\right|^{2}+\left|u^{\prime}\left(t, z, \frac{j \pi}{p}\right)\right|^{2} d z d t .
$$

We will carry out the proof without using the condition $p=q$, obtaining the estimate

$$
c E \leq \sum_{j=1}^{p-1} \int_{-R}^{R} \int_{0}^{\pi}\left|u^{\prime}\left(t, j \pi / p, x_{2}\right)\right|^{2} d x_{2} d t+\sum_{j=1}^{q-1} \int_{-R}^{R} \int_{0}^{\pi}\left|u^{\prime}\left(t, x_{1}, j \pi / q\right)\right|^{2} d x_{1} d t
$$


if $R$ is sufficiently large. However, for $p \neq q$ the condition (9) is replaced by a more complicated expression, difficult to evaluate (see (10) below).

For the proof we start by deducing from (6) that

$$
u^{\prime}\left(t, x_{1}, x_{2}\right)=\sum_{k_{1}=1}^{\infty} \sum_{k_{2}=1}^{\infty} i|k|\left(a_{k} e^{i|k| t}-b_{k} e^{-i|k| t}\right) \sin \left(k_{1} x_{1}\right) \sin \left(k_{2} x_{2}\right) .
$$

Hence for each $j=1, \ldots, p-1$ we have

$$
\begin{aligned}
& u^{\prime}\left(t, j \pi / p, x_{2}\right)=\sum_{k_{1}=1}^{\infty} \sum_{k_{2}=1}^{\infty} i|k|\left(a_{k} e^{i|k| t}-b_{k} e^{-i|k| t}\right) \sin \left(k_{1} j \pi / p\right) \sin \left(k_{2} x_{2}\right)= \\
& =\sum_{\ell=1}^{2 p} \sin (\ell j \pi / p) \sum_{k_{1} \equiv \ell} \sum_{k_{2}=1}^{\infty} i|k|\left(a_{k} e^{i|k| t}-b_{k} e^{-i|k| t}\right) \sin \left(k_{2} x_{2}\right) ;
\end{aligned}
$$

in the middle sum of the last line $k_{1}$ runs over the set of positive integers congruent to $\ell$ modulo $2 p$.

Since $\sin (\ell(j+p) \pi / p)=(-1)^{\ell} \sin (\ell j \pi / p)$ for $j=1, \ldots, p$, we may rewrite the last equality as

$$
u^{\prime}\left(t, j \pi / p, x_{2}\right)=\sum_{\ell=1}^{p} \sin (\ell j \pi / p) f_{\ell}\left(t, x_{2}\right)
$$

with

$$
f_{\ell}\left(t, x_{2}\right)=i \sum_{k_{1} \equiv \ell} \sum_{k_{2}=1}^{\infty} \pm|k|\left(a_{k} e^{i|k| t}-b_{k} e^{-i|k| t}\right) \sin \left(k_{2} x_{2}\right) ;
$$

here and in the following formulas $k_{1}$ runs over the set of positive integers congruent to $\ell$ modulo $p$ (and not $2 p) .{ }^{5}$

Since $\sin (\ell j \pi / p)=0$ for $j=p$ and since the matrix $(\sin (\ell j \pi / p))_{\ell, j=1}^{p-1}$ is invertible ${ }^{6}$, it follows that

$$
c_{1} \sum_{\ell=1}^{p-1}\left|f_{\ell}\left(t, x_{2}\right)\right|^{2} \leq \sum_{j=1}^{p-1}\left|u^{\prime}\left(t, \ell j \pi / p, x_{2}\right)\right|^{2} \leq c_{2} \sum_{\ell=1}^{p-1}\left|f_{\ell}\left(t, x_{2}\right)\right|^{2}
$$

with two positive constants $c_{i}=c_{i}(p), i=1,2$.

Integrating the left inequality and using the orthogonality of the functions $\sin \left(k_{2} x_{2}\right)$ we get

$$
\begin{aligned}
& \sum_{j=1}^{p-1} \int_{0}^{\pi}\left|u^{\prime}\left(t, \ell j \pi / p, x_{2}\right)\right|^{2} d x_{2} \geq c_{1} \sum_{\ell=1}^{p-1} \int_{0}^{\pi}\left|f_{\ell}\left(t, x_{2}\right)\right|^{2} d x_{2}= \\
& =\frac{c_{1} \pi}{2} \sum_{\ell=1}^{p-1} \sum_{k_{2}=1}^{\infty}\left|\sum_{k_{1} \equiv \ell} \pm\right| k\left|\left(a_{k} e^{i|k| t}-b_{k} e^{-i|k| t}\right)\right|^{2} .
\end{aligned}
$$

\footnotetext{
${ }^{5}$ The signs \pm can be precised, but it is not necessary for the sequel: it is equal to 1 if $k_{1} \equiv \ell \bmod 2 p$, and -1 if $k_{1} \equiv \ell+p \bmod 2 p$.

${ }^{6}$ See [16, Problem 277].
} 
Integrating in time the preceding inequality, using Lemma 1 and observing that now we may apply Theorem 1 with $\gamma_{1}=(\sqrt{2}-1) p$ (instead of $\gamma_{1}=(\sqrt{2}-1)$ ) for each fixed $k_{2}$ because of the congruence condition $k_{1} \equiv \ell \bmod p$, we obtain the following estimate:

$$
\begin{aligned}
& \sum_{j=1}^{p-1} \int_{-R}^{R} \int_{0}^{\pi}\left|u^{\prime}\left(t, \ell j \pi / p, x_{2}\right)\right|^{2} d x_{2} d t \geq \\
& \quad \geq \frac{c_{1} \pi}{2} \sum_{\ell=1}^{p-1} \sum_{k_{2}=1}^{\infty} \frac{4 R}{\pi}\left(\sum_{\substack{k_{1} \equiv \ell \\
k_{1} \geq k_{2}}}|k|^{2}\left(\left|a_{k}\right|^{2}+\left|b_{k}\right|^{2}\right)-\frac{\pi^{2}}{R^{2} \gamma_{1}^{2}} \sum_{k_{1} \equiv \ell}|k|^{2}\left(\left|a_{k}\right|^{2}+\left|b_{k}\right|^{2}\right)\right) .
\end{aligned}
$$

Since for $(p, q)$-cyclic solutions we have $a_{k}=b_{k}=0$ whenever $k_{1} \equiv 0$ (see [11, Lemma 4.1]), we may change $\sum_{\ell=1}^{p-1}$ artificially to $\sum_{\ell=1}^{p}$. Then the last expression becomes

$$
\begin{aligned}
& \sum_{j=1}^{p-1} \int_{-R}^{R} \int_{0}^{\pi}\left|u^{\prime}\left(t, \ell j \pi / p, x_{2}\right)\right|^{2} d x_{2} d t \geq \\
& \quad \geq 2 c_{1} R \sum_{k_{2}=1}^{\infty}\left(\sum_{k_{1}=k_{2}}^{\infty}|k|^{2}\left(\left|a_{k}\right|^{2}+\left|b_{k}\right|^{2}\right)-\frac{\pi^{2}}{R^{2} \gamma_{1}^{2}} \sum_{k_{1}=1}^{\infty}|k|^{2}\left(\left|a_{k}\right|^{2}+\left|b_{k}\right|^{2}\right)\right) .
\end{aligned}
$$

Exchanging the role of $x_{1}$ and $x_{2}$ we have also the symmetric estimate

$$
\begin{aligned}
& \sum_{j=1}^{q-1} \int_{-R}^{R} \int_{0}^{\pi}\left|u^{\prime}\left(t, x_{1}, \ell j \pi / q\right)\right|^{2} d x_{1} d t \geq \\
& \quad \geq 2 c_{1} R \sum_{k_{1}=1}^{\infty}\left(\sum_{k_{2}=k_{1}}^{\infty}|k|^{2}\left(\left|a_{k}\right|^{2}+\left|b_{k}\right|^{2}\right)-\frac{\pi^{2}}{R^{2} \gamma_{2}^{2}} \sum_{k_{2}=1}^{\infty}|k|^{2}\left(\left|a_{k}\right|^{2}+\left|b_{k}\right|^{2}\right)\right)
\end{aligned}
$$

with $\gamma_{2}=(\sqrt{2}-1) q$. Adding the two inequalities we conclude that

$$
\begin{array}{r}
\sum_{j=1}^{p-1} \int_{-R}^{R} \int_{0}^{\pi}\left|u^{\prime}\left(t, \ell j \pi / p, x_{2}\right)\right|^{2} d x_{2} d t+\sum_{j=1}^{q-1} \int_{-R}^{R} \int_{0}^{\pi}\left|u^{\prime}\left(t, x_{1}, \ell j \pi / q\right)\right|^{2} d x_{1} d t \\
\quad \geq c \sum_{k_{1}=1}^{\infty} \sum_{k_{2}=1}^{\infty}|k|^{2}\left(\left|a_{k}\right|^{2}+\left|b_{k}\right|^{2}\right)
\end{array}
$$

with

$$
c:=2 R\left(\min \left\{c_{1}(p), c_{1}(q)\right\}-\frac{\pi^{2} c_{1}(p)}{R^{2} \gamma_{1}^{2}}-\frac{\pi^{2} c_{1}(q)}{R^{2} \gamma_{2}^{2}}\right) .
$$

In view of (7) the theorem follows if $c>0$. For $p=q$ this is equivalent to (9).

Part of this work was done during the visit of the first author at the Dipartimento di Scienze di Base e Applicate per l'Ingegneria of the Sapienza Università di Roma in May-July 2014. The author wishes to thank the department for its hospitality. 


\section{References}

1. Lasiecka I., Triggiani R. Regularity of Hyperbolic Equations under $L_{2}\left(0, T ; L_{2}(\Gamma)\right)$ Boundary Terms. Appl. Math. and Optimiz., 1983, vol. 10, pp. 275-286. DOI: 10.1007/BF01448390

2. Lions J.-L. Contrôle des systèmes distribués singuliers. Paris, Gauthier-Villars, 1983.

3. Ho L.F. Observabilité frontière de l'équation des ondes. C. R. Acad. Sci. Paris Sér. I Math., 1986, vol. 302, pp. 443-446.

4. Lions J.-L. Exact Controllability, Stabilizability, and Perturbations for Distributed Systems. Siam Rev., 1988, vol. 30, pp. 1-68. DOI: 10.1137/1030001

5. Lions J.-L. Contrôlabilité exacte et stabilisation de systèmes distribués I-II. Masson, Paris, 1988.

6. Bardos C., Lebeau G., Rauch J. Sharp Sufficient Conditions for the Observation, Control and Stabilization of Waves from the Boundary. SIAM J. Control Optim., 1992, vol. 30, pp. 1024-1065. DOI: $10.1137 / 0330055$

7. Komornik V. Controlabilité Exacte en un Temps Minimal. C. R. Acad. Sci. Paris Sér. I Math., 1987, vol. 304, pp. 223-225.

8. Mehrenberger M. An Ingham Type Proof for the Boundary Observability of a N-d Wave Equation. C. R. Math. Acad. Sci. Paris, 2009, vol. 347, no. 1-2, pp. 63-68. DOI: $10.1016 /$ j.crma.2008.11.002

9. Ingham A.E. Some Trigonometrical Inequalities with Applications in the Theory of Series. Math. Z., 1936, vol. 41, pp. 367-379. DOI: 10.1007/BF01180426

10. Komornik V., Miara B. Cross-Like Internal Observability of Rectangular Membranes. Evol. Equations and Control Theory, 2014, vol. 3, no. 1, pp. 135-146. DOI: 10.3934/eect.2014.3.135

11. Komornik V., Loreti P. Observability of Rectangular Membranes and Plates on Small Sets. Evol. Equations and Control Theory, 2014, vol. 3, no. 2, pp. 287-304. DOI: $10.3934 /$ eect.2014.3.287

12. Komornik V., Loreti P. Fourier Series in Control Theory. New York, Springer-Verlag, 2005.

13. Gasmi S., Haraux A. N-Cyclic Functions and Multiple Subharmonic Solutions of Duffing's Equation. J. Math. Pures Appl., 2012, vol. 97, pp. 411-423. DOI: $10.1016 /$ j.matpur.2009.08.005

14. Baiocchi C., Komornik V., Loreti P. Ingham Type Theorems and Applications to Control Theory. Bol. Un. Mat. Ital. B. Series 8, 1999, vol. 2, no. 1, pp. 33-63.

15. Baiocchi C., Komornik V., Loreti P. Ingham-Beurling Type Theorems with Weakened Gap Conditions. Acta Math. Hungar., 2002, vol. 97, no. 1-2, pp. 55-95. DOI: $10.1023 / \mathrm{A}: 1020806811956$

16. Fadeev D.K., Sominsky D.K. Problems in Higher Algebra. Moscow, Mir, 1972.

17. Haraux A. Séries lacunaires et controle semi-interne des vibrations d'une plaque rectangulaire. J. Math. Pures Appl., 1989, vol. 68, pp. 457-465.

18. Komornik V., Loreti P. Ingham Type Theorems for Vector-Valued Functions and Observability of Coupled Linear Systems. SIAM J. Control Optim., 1998, vol. 37, pp. 461-485. DOI: $10.1137 / \mathrm{S} 0363012997317505$

19. Komornik V., Loreti P. Multiple-Point Internal Observability of Membranes and Plates. Appl. Anal., 2011, vol. 90, no. 10, pp. 1545-1555. DOI: 10.1080/00036811.2011.569497

20. Loreti P. On Some Gap Theorems. Proceedings of the 11th Meeting of EWM, CWI Tract, 2005. 
21. Loreti P., Mehrenberger M. An Ingham Type Proof for a Two-Grid Observability Theorem ESAIM Control Optim. Calc. Var., 2008, vol. 14, no. 3, pp. 604-631.

22. Loreti P., Valente V. Partial Exact Controllability for Spherical Membranes. SIAM J. Control Optim., 1997, vol. 35, pp. 641-653. DOI: 10.1137/S036301299526962X

Vilmos Komornik, Département de mathématique, Université de Strasbourg, 7 rue René Descartes, 67084 Strasbourg Cedex, France, vilmos.komornik@math.unistra.fr.

Paola Loreti, Dipartimento di Scienze di Base e Applicate per l'Ingegneria, Sapienza Università di Roma, via A. Scarpa n. 16, 00161 Roma, Italia, paola.loreti@sbai.uniroma1.it.

\title{
НАБЛЮДАЕМОСТЬ КВАДРАТНЫХ МЕМБРАН МЕТОДАМИ РЯДОВ ФУРЬЕ
}

\section{В. Коморник, П. Лорети}

\begin{abstract}
Долгое время в теории управления успешно применялись методы Фурье. Однако для некоторых теорем этот подход не применим. Несколько лет назад Мегренберг установил граничную наблюдаемость колебаний прямоугольной мембраны (и для аналогичных задач большей размерности), обобщив классическую теорему Ингама о негармонических рядах Фурье. Предложенный им метод оказался полезным и для других приложений. Мы совершенствуем подход Мегренберга, сократив доказательство, а также обобщаем некоторые ранее рассмотренные приложения.

Ключевые слова: наблюдаемость; негармонический ряд Фуръе; теорема Ингама; волновое уравнение.
\end{abstract}

Вилмос Коморник, профессор, Страсбургский университет (г. Страсбург, Франция), vilmos.komornik@math.unistra.fr.

Паола Лорети, профессор, Римский университет Ла Сапиенца (г. Рим, Италия), paola.loreti@sbai.uniroma1.it.

Поступила в редакиию 11 декабря 2014 г. 